\title{
Is post-surgical rehabilitation sufficient? Would it be better to rehabituate?
}

\author{
John Nyland ${ }^{1}$
}

Published online: 25 July 2018

(c) European Society of Sports Traumatology, Knee Surgery, Arthroscopy (ESSKA) 2018

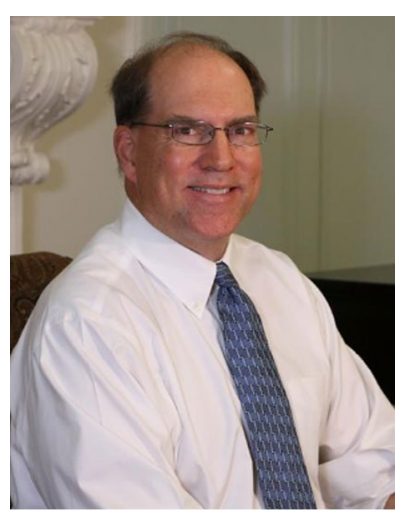

John Nyland

To rehabilitate someone... what does that mean? Does it mean eventual complete recovery in the sense that all is returned to the pre-morbid condition? Occasionally, this may indeed be the case. However, this expectation may more likely be achieved only when natural tissue healing or primary anatomic surgical repair occurs and when the final healing stages closely mirror that of the native tissues.

Following joint preserving ligament reconstruction and arthroplasty, however, natural tissue healing is not restored making this goal somewhat moot. Following ligament reconstruction and arthroplasty, rehabilitation expectations may need to differ compared to natural tissue healing or primary anatomic surgical repair. This is because the tissue remodelling that occurs in both cases differs considerably from native tissues histologically, physiologically and biomechanically, and in the case of arthroplasty, living tissues are replaced with a combination of plastic and/or metal parts.

John Nyland

jnyland@ spalding.edu

1 Kosair Charities College of Health and Natural Sciences, Spalding University, 901 South 4th Street, Louisville, KY 40203, USA
Since these surgically reconstructed or replaced tissues possess widely diverse characteristics, one might surmise that patient outcome goals (both surgical and rehabilitative) may also differ somewhat. Within this context, variable long-term strength/power, neuromuscular control, fall reduction, or pain management coping skills training may need to continue to promote sustainable functional recovery, surgical failure prevention, contralateral joint or adjacent tissue injury prevention, positive health behaviour enhancement and improved health-related quality of life. With this ongoing therapeutic intervention, the patient may successfully avoid the need for further surgery or rehabilitation. This indeed is a lofty, and perhaps unrealistic goal. However, it also represents a large portion of the benefits that physiotherapists with contributions from other professionals such as nutritionists, strength coaches, athletic trainers, nurses, sports psychologists and others are uniquely trained to provide $[1,2]$. If these rehabilitative needs are not provided by this inter-professional team, the stage may be set for the patient to experience a future of additional injuries, further surgery and/or rehabilitation, unfulfilled expectations and a suddenly steeper downward spiral toward greater impairments, functional limitations, disabilities and a reduced health-related quality of life. If all needs are not met during rehabilitation, this downward spiral may represent the future of many unfortunate patients following joint reconstruction and arthroplasty. In addition to appropriate patient-surgical procedure matching, surgical timing and rehabilitation approach selection, education both before and after surgery, is essential to optimal recovery [6].

The word "habituate" means to make fit or capable for functioning in society [3]. It also has been defined as the capacity to equip or to be made able, to transform or change the state of, to make suitable for, to enable, qualify, allow, empower or prepare, to develop important life skills [4]. I submit that it is essential that physiotherapists reflect about their capacity to independently fulfill the comprehensive expanse of patient needs following joint reconstruction and 
arthroplasty for activities beyond basic activities of daily living. While the first part may be achieved relatively quickly, the latter requires steadfast attention to a plethora of interacting complexities, warranting validated criteria to map and monitor recovery plan success. To effectively restore activity independence beyond this, such as for athletic or sports role restoration, greater inter-professional team involvement may be needed to adequately accommodate individual patient needs. This becomes particularly true when one considers that these unfulfilled needs often exist long after the time period allotted to professional physiotherapy services by third party payers [5].

To achieve effective post-surgical and rehabilitation outcomes at 2 (early), 5 (intermediate) and more than 10 years (long-term) post-intervention warrants a better understanding of the potential influence of all others who interact with the patient and the activities that the patient engages in over these extended time periods [1,2]. Although the physiotherapist may no longer be regularly treating the patient at this time, they should continue to interact with them at least from a consultatory or advisory perspective. Depending on the unique characteristics of each patient, the knowledge base and opinions of a wide range of diverse professionals and associated others may also need to be sought and obtained.

The physiotherapist may be uniquely skilled in overseeing the process of ensuring that the patient becomes accustomed, adjusted or acclimated to future impactful life situations [1, 2]. By necessity, the patient recovery focus should evolve from comparatively shorter term, relatively quicker basic activity of daily living achievement to the successful achievement of longer term rehabituation goals. Instead of simply focusing on returning the patient to sports as quickly as possible, perhaps greater focus should be placed on helping them develop the steadfast perseverance and coping abilities necessary to minimise and manage future risks over a longer recovery time period [5]. Given the growing influence of health-related quality of life and patient satisfaction outcomes data on practitioner and hospital performance rankings, should not successful rehabituation become the ultimate goal for both surgical and rehabilitative interventions? Innovative surgical approaches, instrumentation designs and material options continues to reduce procedural invasiveness further decreasing tissue morbidity. Associated with this, patients are encouraged to mobilise, weight bear as tolerated and become more active earlier during the recovery process. Rehabilitation needs to similarly evolve with greater appreciation for the importance of inter-professional teams in helping patients safely return to their desired role, becoming successfully rehabituated.

\section{References}

1. Ardern CL, Grindem H, Ekås GR, Seil R, McNamee M (2018) Applying ethical standards to guide shared decision-making with youth athletes. Br J Sports Med 49:865-870. https://doi. org/10.1136/bjsports-2018-099183

2. Goldring AE, Ashok AP, Casey EK, Mulcahey MK (2016) Key components and potential benefits of a comprehensive approach to women's musculoskeletal health. Phys Sportsmed 44:417-424

3. http://www.dictionary.com/browse/habituate. Retrieved 9 July 2018

4. https://www.merriam-webster.com/dictionary/habituate. Retrieved 9 July 2018

5. Nagelli CV, Hewett TE (2017) Should return to sport be delayed until 2 years after anterior cruciate ligament reconstruction? Biological and functional considerations. Sports Med 47(2):221-232

6. Tingle C (2018) Education, strength training important for return to sport after ACL reconstruction. https://www.healio.com/ortho pedics/total-joint-reconstruction/ news/online/\% 7bf6fa2064-be394381-a044-2c98ff30c8da\%7d/education-strength-training-impor tant-for-return-to-sport-after-acl-reconstruction. Accessed 9 July 2018 